\title{
Bilateral synchronous ovarian tumours: an uncommon case and review of the literature
}

\author{
Emre Günakan¹, Yusuf A. Tohma², Asuman N. Haberal' ${ }^{3}$ Ali Ayhan² \\ ${ }^{1}$ Department of Obstetrics and Gynecology, Keçiören Training and Research Hospital, Ankara, Turkey \\ 2Department of Obstetrics and Gynecology, Baskent University School of Medicine, Ankara, Turkey \\ ${ }^{3}$ Department of Pathology, Baskent University School of Medicine, Ankara, Turkey
}

\begin{abstract}
Synchronous ovarian tumours are rare. Management of these patients can differ from that of patients with uniform tumours. We present a case of synchronous epithelial ovarian cancer and malignant mixed Müllerian tumour in different ovaries, its follow-up and management until death. To our knowledge this is the second case in the English literature to date.

A 61-year-old woman with bilateral adnexal masses underwent complete debulking surgery for ovarian cancer. The final pathology was reported as malignant mixed Müllerian tumour in the right ovary with intact borders and stage 2 grade 3 serous carcinoma in the left ovary. She had a 17-month disease-free interval after 6 cycles of paclitaxel and carboplatin. Recurrence of malignant mixed Müllerian tumour was reported in the pathology after secondary debulking including a partial ileal resection. After 6 cycles of gemcitabine and cisplatin she had a widespread recurrence in the thorax and abdomen. The patient died of disease recurrence at the $25^{\text {th }}$ month after diagnosis.

Coexistence of serous and malignant mixed Müllerian tumour in different ovaries is very rare. The main treatment is complete cytoreduction followed with chemotherapy. Platinum-taxane based chemotherapy resulted in an acceptable disease-free interval in our case, but it is not standard yet. A management protocol may be developed with the increasing number of similar cases in the literature.
\end{abstract}

Key words: synchronous ovarian tumours, serous adenocarcinoma, malignant mixed Müllerian tumour.

\section{Introduction}

In the literature coexistence of ovarian and endometrial cancers is the most common in synchronous genital tract cancers. Bilateral ovarian tumours are extremely rare, and therefore limited data are available for the management of these cases.

Epithelial ovarian cancer (EOC) is the most common in ovarian cancers and accounts for nearly $90 \%$ of all [1]. The majority of cases are diagnosed at an advanced stage and frequently with bilateral ovarian and peritoneal invasion. Ovarian malignant mixed Müllerian tumours (MMMT) are very rare and account for only $1-4 \%$ of ovarian cancers [2].

Both EOC and ovarian MMMT usually present with an adnexal mass and advanced stage disease [3]. Also both have similar management including debulking surgery and concomitant chemotherapy. Cytoreductive surgery is the initial approach for both in advanced stages $[4,5]$. Platin-based chemotherapy is standard for EOC in first-line therapy [6] and also has a positive prognostic effect in MMMT [7].
We present a case with serous type EOC and MMMT, diagnosed at two different ovaries, and the issue is discussed together with the literature.

\section{Case presentation}

A 61-year-old postmenopausal woman was referred to our clinic with a pelvic mass. Her primary symptom was pelvic pain. Bilateral adnexal masses and pelvic fluid collection were determined with pelvic examination and transvaginal ultrasonography. In laboratory findings, Ca-125 concentration was $350.7 \mathrm{U} / \mathrm{ml}$. Thorax and abdominal computed tomography also indicated an ovarian neoplasm limited to the pelvis.

In the light of these findings the patient was subjected to laparotomy with a midline incision. Hysterectomy and bilateral salpingoophorectomy material was sent for frozen section and reported as a malign neoplasm of the ovary. Staging surgery was performed for ovarian cancer (Fig. 1). The final pathology was reported as MMMT (carcinoma component serous + endome- 


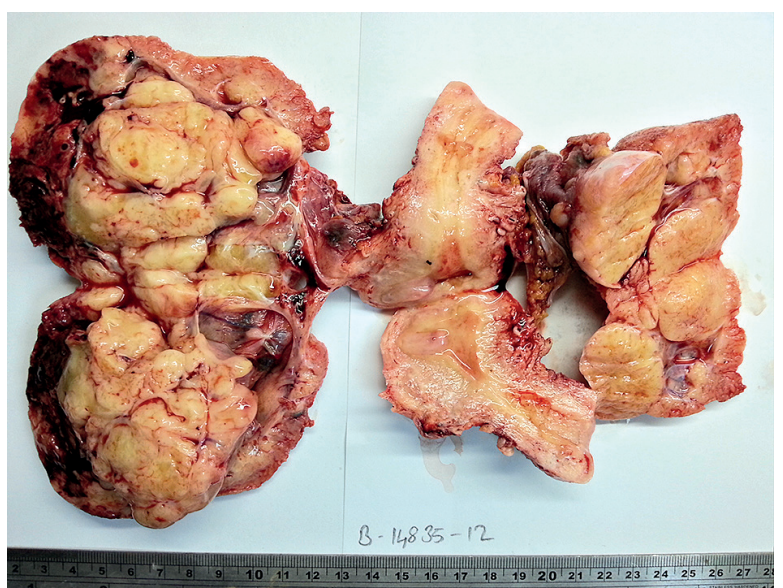

Fig. 1. TAH + BSO gross view. Normal uterus. Right ovary has multilobulated solid and haemorrhagic areas, and left ovary has a yellow-grey solid + cystic pattern

trioid and sarcomatous component with heterologous elements of chondrosarcoma and rhabdomyosarcoma) in the right ovary with intact borders and grade 3 serous carcinoma in the left ovary, with a tumour on the surface (Figs. 2 and 3). Abdominal washing was tumour positive. None of 49 lymph nodes was involved but necrotic carcinosarcomatous tumour nodules were seen in the left external and obturator region. There were no complications in the postoperative period and the patient was discharged on the $7^{\text {th }}$ day. She was discussed in our gynaecology oncology unit and 6 cycles of paclitaxel + carboplatin every 21 days treatment was planned. Her treatment was performed regularly with no adverse affects of chemotherapy and finished in the $4^{\text {th }}$ month after surgery. In the follow-up, she had a recurrence at the 17th month in April 2014 and was subjected to debulking surgery with colonic resection. The final pathology was reported as recurrence of MMMT. Gemcitabine + cisplatin chemotherapy every 21 days was planned again. After regular treatment finished at

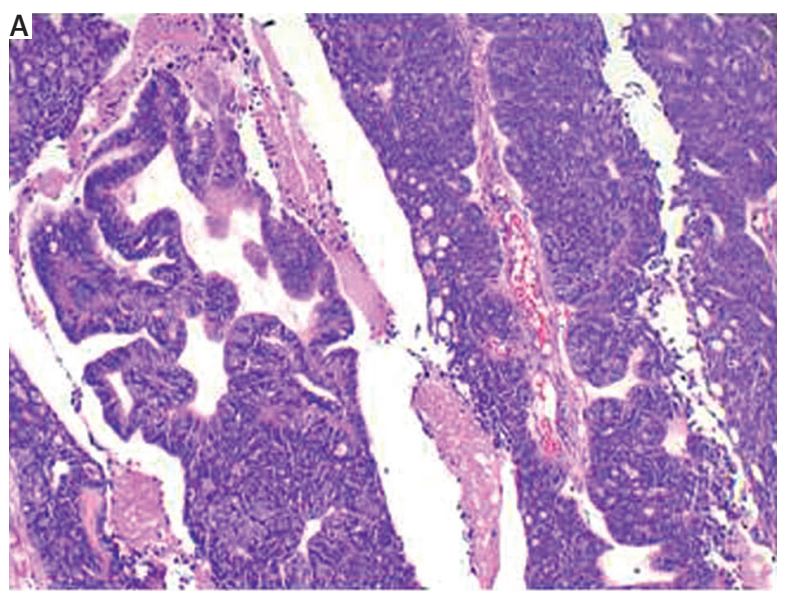

4 months she had widespread disease in two months and despite intensive care she died in the $25^{\text {th }}$ month after the first diagnosis of the disease.

\section{Discussion}

EOCs are the most common malignancies of the ovary. Serous histology is the most common type and is generally caught at advanced stages and bilateral ovarian involvement.

Otherwise MMMT is a rare ovarian tumour which occurs from pluripotent mesenchymal cells of the coelomic epithelium. These are differentiated carcinomas of the ovary and are thought to occur from pre-existing carcinomas.

Bilateral ovarian synchronous tumours are very rare. Bernárdez Zapata et al. presented a 49-year-old patient with two different epithelial cancers in two ovaries: a clear cell carcinoma in the left ovary, and a moderately differentiated endometrioid carcinoma in the right one [8]. Semczuk et al. reported a 73 year-old patient with synchronous granulosa cell tumour and a fibroma [9].

Epithelial and sarcomatous synchronous neoplasms have different origins and are more uncommon. Only one case similar to ours has been described before a 58-year-old woman with bilateral ovarian synchronous malignant tumours [10]. In 2008, ovarian cancer with a mesenteric MMMT was presented by Ma et al. [11]. Arora et al. reported a case of MMMT of the broad ligament in association with uterine endometrioid adenocarcinoma along with papillary serous carcinoma of the ovary [12]. Tae Yeon Lee et al. presented a 57-year-old patient with MMMT that originated from both the uterus and ovary [13].

EOC and MMMT are usually diagnosed at advanced stages and have a poor prognosis. Stage and optimal cytoreductive surgery are important prognostic factors for both. These cancers have highly aggressive natures

Fig. 2. Histopathological image of left ovary. Serous adenocarcinoma (H\&E $\times 10$ original magnification and inset $\times 20$ original magnification)

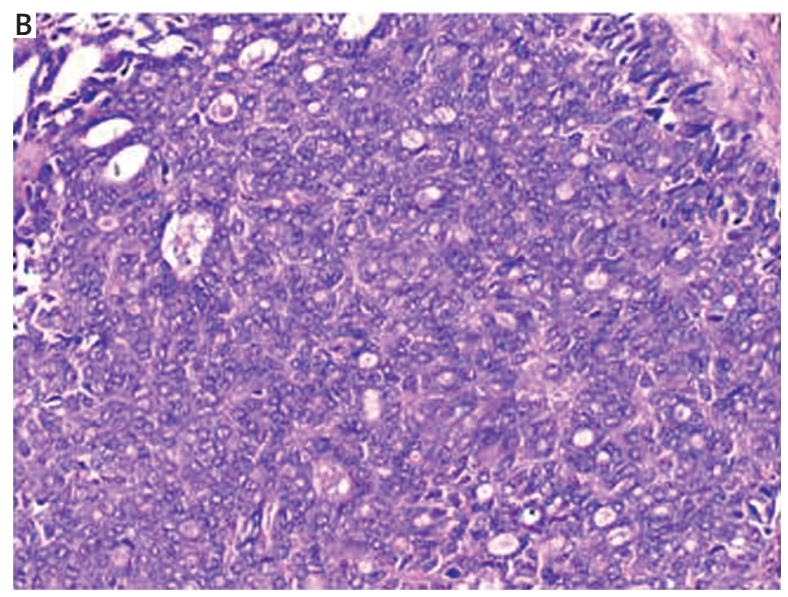



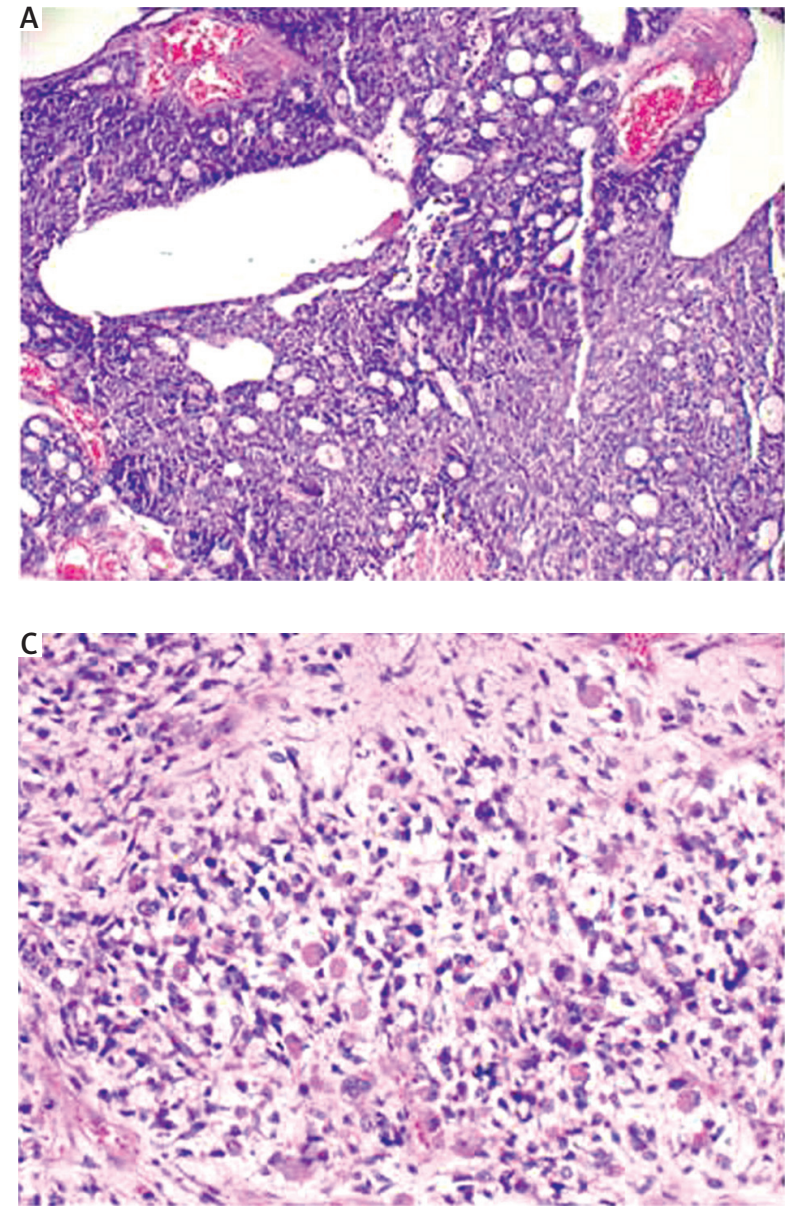

and in management after debulking and staging surgery, chemotherapy is needed. Platinum-taxane based chemotherapy is the first choice for EOC. Multiple adjuvant therapies for MMMT have been reported in the literature including doxorubicin, cisplatin, dacarbazine, paclitaxel, carboplatin and ifosfamide [14-16]. In terms of these approaches platinum-taxane based adjuvant therapy may be a good option for synchronised MMMT and EOC.

\section{Conclusions}

We have reported the case of a patient with serous adenocarcinoma and MMMT in different ovaries from the first diagnosis until death. MMMT was stage $2 b$ and serous cancer was stage 1c. The patient was treated with paclitaxel and carboplatin chemotherapy, which is standard for ovarian cancer and well accepted for MMMT treatment. After treatment she had a 17-month disease-free survival.

Today there is an increasing trend in synchronous tumour cases. There have been reports with simultaneous occurrence of two different tumours at different sites in the female genital tract. These may have more complicated management or treatment. We believe

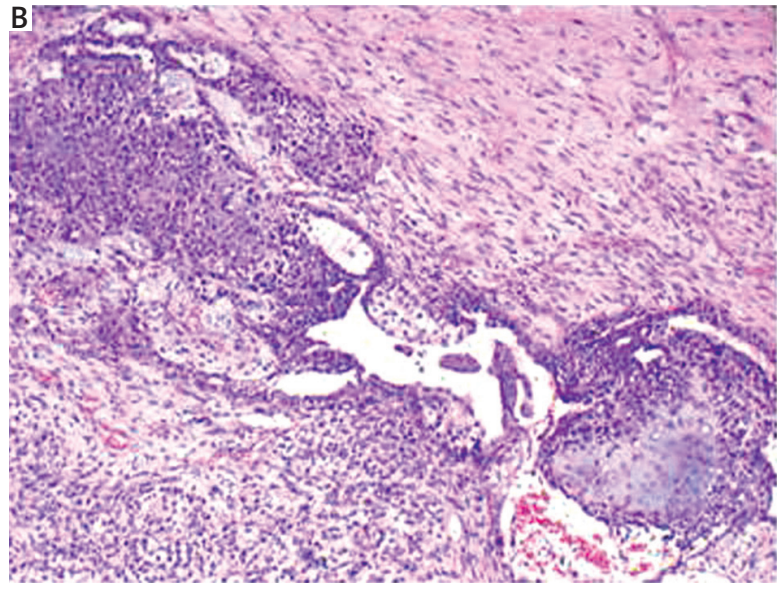

Fig. 3. Histopathological image of right ovary. Epithelial adenocarcinoma component, rhabdomyosarcomatous and chondrosarcomatous heterologous elements of malignant mixed Müllerian tumours (H\&E $\times 10$ original magnification)

that platinum-taxane based chemotherapy may be a good choice in patients with synchronous EOC and MMMT after optimal cytoreduction. Therefore, sharing more knowledge and more cases will be advantageous. Here we have reported a case of serous tumour and MMMT in two ovaries with their management to contribute to the literature.

\section{Disclosure}

The authors report no conflict of interests.

\section{References}

1. Reid BM, Permuth JB, Sellers TA. Epidemiology of ovarian cancer: a review. Cancer Biol Med 2017; 14: 9-32.

2. del Carmen MG, Birrer M, Schorge JO. Clear cell carcinoma of the ovary: a review of the literature. Gynecol Oncol 2012; 126: 481-490.

3. Mara R (ed.).Epithelial carcinoma of the ovary, fallopian tube, and peritoneum: Histopathology. Post TW, ed. UpToDate. Waltham, MA: UpToDate Inc. http://www.uptodate.com (Accessed on February 10, 2018.)

4. Chang SJ, Hodeib M, Chang J, Bristow RE. Survival impact of complete cytoreduction to no gross residual disease for advanced-stage ovarian cancer: a meta-analysis. Gynecol Oncol 2013; 130: 493-498.

5. Navarini R, Pineda RL. Malignant mixed müllerian tumors of the ovary. Curr Opin Obstet Gynecol 2006; 18: 20-23.

6. Bookman MA, Greer BE, Ozols RF. Optimal therapy of advanced ovarian cancer: carboplatin and paclitaxel vs. cisplatin and paclitaxel (GOG 
158) and an update on GOGO 182-ICON5. Int J Gynecol Cancer 2003; 13: 735-740.

7. Mano MS, Rosa DD, Azambuja E, et al. Current management of ovarian carcinosarcoma. Int J Gynecol Cancer 2007; 17: 316-324.

8. Bernárdez Zapata F, Jáuregui Meléndrez RA, Cabrera Carranco E. Synchronous double primary ovarian tumor in situ. Ginecol Obstet Mex 2012; 80: 480-486.

9. Semczuk A, Skomra D, Gogacz M, et al. Synchronous adult-type granulosa cell tumor of the ovary with ovarian fibroma: a case report. Eur J Gynaecol Oncol 2011; 32: 691-694.

10. Song MJ, Lee CW, Seo KJ, et al. A case of bilateral ovarian synchronous tumors (left ovarian serous papillary adenocarcinoma and right ovarian malignant mixed Müllerian tumor). Eur J Gynaecol Oncol 2011; 32: 234-236.

11. Ma CJ, Yang SF, Huang CC, et al. Malignant mixed müllerian tumor of primary mesenteric origin associated with a synchronous ovarian cancer: case report and literature review. Eur J Gynaecol Oncol 2008; 29: 289-293.

12. Arora P, Rao S, Khurana N, et al. Malignant mixed Mullerian tumor of broad ligament with synchronous ovarian and endometrial carcinoma: a rare association. J Cancer Res Ther 2011; 7: 88-91.

13. Lee TY, Lee Ch, Choi WJ, et al. Synchronous occurrence of primary malignant mixed müllerian tumor in ovary and uterus. Obstet Gynecol Sci 2013; 56: 269-272

14. Baker TR, Piver MS, Caglar H, Piedmonte M. Prospective trial of cisplatin, adriamycin, and dacarbazine in metastatic mixed mesodermal sarcomas of the uterus and ovary. Am J Clin Oncol 1991; 9: 246.

15. Kosmas C, Vorgias G, Tsakonas G, et al. Paclitaxel-ifosfamide-carboplatin combination chemotherapy regimen in advanced uterine and adnexal malignantmixed Mullerian tumours. Br J Cancer 2011; 105: 897-902.

16. Powell MA, Filiaci VL, Rose PG, et al. Phase II evaluation of paclitaxel and carboplatin in the treatment of carcinosarcoma of the uterus: a Gynecologic Oncology Group study. J Clin Oncol 2010; 28: 2727-2731. 